\title{
Understanding macroscopic thermodynamic properties of hydrothermal fluids from molecular dynamics simulations
}

\author{
MAXIMILIAN SCHULZE AND SANDRO JAHN
}

Institute of Geology and Mineralogy, University of Cologne

Presenting Author: maximilian.schulze@uni-koeln.de

The mechanisms behind fluid-rock interactions play a critical role in understanding mass transfer in a variety of geological environments. Particularly in the georesources area, insights into the transport behavior of aqueous solutes and the precipitation of minerals are of great interest. For example, the economic profitability of geothermal exploitation depends heavily on the formation of scaling in wells and the corrosive character of the fluids extracted.

Our quantitative understanding of such phenomena is largely based on the application of thermodynamic modeling approaches. However, none of the existing equations of state (EoS) that can treat the thermodynamics of ions and molecules in aqueous solutions is universally applicable to the entire spectrum of geological pressure and temperature conditions. The widely utilized revised Helgeson-Kirkham-Flowers model [1], for instance, cannot be used near the critical point conditions of water.

Our aim is to link macroscopic thermodynamic quantities with the underlying properties at the atomic scale. This will provide a molecular based guideline for the future development of new EoS. For this purpose we perform classical molecular dynamics simulations (MD) of the model system $\mathrm{NaCl}-\mathrm{H}_{2} \mathrm{O}$. In our preliminary work we obtained dissociation constants of $\mathrm{NaCl}$ in dilute aqueous solutions at temperatures above $400^{\circ} \mathrm{C}$ utilizing $\mathrm{MD}$ in conjunction with metadynamics [2]. This approach confirms linear dependencies between the logarithm of the dissociation constant and the logarithm of water density that are known from experiments [3]. Further investigations will focus on the relationships between fluid structure and thermodynamic quantities such as equilibrium constants and partial molar volumes.

This work was funded within EU2020 project GEOPRO, grant agreement ID 851816.

[1] Helgeson HC, Kirkham DH, Flowers GC (1981) Theoretical prediction of the thermodynamic behavior of aqueous electrolytes at high pressures and temperatures. Am J Sci, 281, 1249-1516

[2] Barducci A, Bussi G, Parrinello M (2008) Well-Tempered metadynamics: A smoothly converging and tunable free-energy method. Phys Rev Lett, 100, 020603

[3] Quist AS and Marshall WL (1968) Electrical conductances of aqueous sodium chloride solutions from 0 to $800{ }^{\circ} \mathrm{C}$ and at pressures to 4000 bars. J Phys Chem, 74, 684-703 